\title{
Gallium-67 Uptake in Histological Variants of Non-Hodgkin's Lymphoma - A Correlative Study
}

\author{
Basit Iqbal ${ }^{*}, 1,2$, Geoffrey M Currie ${ }^{2,3}$, Humayun Bashir ${ }^{4}$, Uzma Afzal ${ }^{4}$, M. Khalid Nawaz ${ }^{4}$, \\ Samina Mansoor ${ }^{4}$, Janelle M Wheat ${ }^{2,3}$ and Muhammad Numair Younis ${ }^{5}$
}

\author{
${ }^{I}$ Pakistan Institute of Engineering \& Applied Sciences, Islamabad, Pakistan \\ ${ }^{2}$ Centre for Research in Complex Systems, Charles Sturt University, Australia \\ ${ }^{3}$ Australia School of Advanced Medicine, Macquarie University, Australia \\ ${ }^{4}$ Shaukat Khanum Memorial Cancer Hospital \& Research Center, Lahore, Pakistan \\ ${ }^{5}$ Institute of Nuclear Medicine \& Oncology, Lahore, Pakistan
}

\begin{abstract}
Introduction: The malignant lymphomas are the fifth most common neoplasm in both men and women. Staging, re-staging and treatment response evaluation pose a difficult task in many patients of lymphomas. Histological variants in both Hodgkin's and Non-Hodgkin's types of lymphoma greatly affect the response to treatment and overall prognosis.

Objective: This study was conducted to correlate the degree of abnormal Gallium-67 uptake with the histological variants of non-Hodgkin's lymphoma. This project was a part of the study to see the ability of Gallium-67 scintigraphy to predict the response to chemotherapy early in the course of therapy.

Material \& Methods: 65 patients were included in the study with a mean age of 25 years. All patients had undergone medical imaging, bone marrow biopsy and histopathology.

Results: Sixty-five patients were classified according to the REAL classification. 11 patients were of T-cell type, 50 were of B-cell type and 4 were anaplastic. 46 showed gallium-67 uptake in the lesions, whereas 19 (29.2\%) had no discernable Gallium-67 uptake.

Conclusion: There was no correlation between histological sub-types and Gallium-67 uptake in all sub-types of NHL.
\end{abstract}

Keywords: Non-Hodgkin's Lymphoma, NHL, Gallium-67, Ga-67 Scintigraphy, Lymphoma Imaging.

\section{INTRODUCTION}

Non-Hodgkin's lymphoma (NHL) represents $4 \%$ of all newly diagnosed cancers in United States and the overall age adjusted rate of NHL are increasing by $1-2 \%$ annually since 1990s [1]. NHL is the fifth most common cancer in both men and women and the sixth most common cause of cancer death, accounting for 4 percent of all cancers and 4 percent of all cancer deaths [2]. NHL is a complex malignancy with many different clinical presentations, histologies and varied treatment options depending on the histological type and other prognostic factors [3].

The International Lymphoma Study Group (ILSG) support the use of the Revised European-American Classification (REAL) system where neoplasms are listed as separate clinical entities based on morphologic, immunophenotypic, and on molecular data, and on the identification of a normal cellular counterpart $[4,5]$. This indicates that ancillary studies, such as immunophenotypic studies and cytogenetic/molecular genetic studies, play an increasingly

*Address correspondence to this author at the Pakistan Institute of Engineering \& Applied Sciences, Islamabad, Pakistan; Tel: 612 69334712; Fax: 612 69332835; E-mail: drbasitiqbal@gmail.com important role in the diagnosis and classification of malignant lymphomas [4-6]. Nonetheless, imaging modalities continue to play an important role in the diagnosis and staging of NHL. While anatomical imaging modalities do not provide any information of histological sub-types of the lymphomas, physiological imaging using single photon emission computed tomography (SPECT) and positron emission tomography (PET) provides a broader range of physiological information about tumors which impact on patient management. Indeed, the recent medical literature has extensive reporting of the role of PET in lymphomas. Unfortunately, PET is not universally readily available for clinical use in NHL across both developed and developing economies (e.g. Pakistan, Australia and Canada). Thus, Gallium-67 (Ga-67) citrate scintigraphy continues to play a globally significant role in NHL assessment.

Gallium-67 scintigraphy, owing to fact that Gallium uptake mechanisms are directly related to the molecular basis of imaged pathology (which are quite complex and yet unclear), is capable of providing unique information such as tumor viability, response to therapy and tumor differentiation $[7,8]$. The characteristic histological variations among different sub-types of NHL may, therefore, be rightly assumed to affect the uptake of Gallium and thus may 
provide useful information. This study was conducted to explore the potential correlation between uptake intensity and the histological sub-types of NHL in order to assess the potential for prediction of the response to therapy early in the course of therapy.

\section{MATERIALS AND METHODS}

\section{Study Design}

All patients referred for Ga-67 scintigraphy with a diagnosis of NHL at Shaukat Khanum Memorial Cancer Hospital \& Research Center (SKMCH \& RC) between 1999 and 2007 were recruited into the study. Informed patient consent was obtained. All patients underwent radiological imaging (US/CT/MRI), bone marrow biopsy and histopathological exam (FNA/Core needle biopsy/Open Biopsy). The patient sample was subjected to the following inclusion criteria:

\section{- $\quad$ Any age or sex.}

- Histologically confirmed diagnosis of NHL.

- NHL patients without any documented liver disease and blood chemistry showing normal liver function.

Patients were excluded from the study on the basis of:

- Receiving therapy prior to the baseline Ga-67 scan.

- Patients in whom the NHL sub-type could not be established.

In all cases histological diagnosis along with results of markers were recorded from patients' record.

\section{Patient Imaging}

Standard Ga-67 citrate imaging was performed at 48 hours (and up to 11 days) post IV administration of 185 $\mathrm{MBq}$ of Ga-67 citrate in 65 cases of NHL between 1999 \& 2007 after due preparation as per standard guidelines. A Siemens DICAM single head gamma camera was used. As a general rule, the clinical protocol was adopted which included whole body imaging at 48 hours post IV, planar views of the axilla, the regions of interest and delayed planar images as required. Amongst these, the number of males was $45(69.2 \%)$ and $20(30.8 \%)$ were females. The number from the pediatric group (less than or equal to 18 years of age.) was $30(46.2 \%)$. The average age of the pediatric group (at the time of diagnosis) was 9.88 years \pm 4.64 (range: 3 to 18 years). The average age of the adult population was 40.23 years with a range of 19-73 years.

Data interpretation was carried out by visual interpretation of images by a group of nuclear physicians during a typical reporting session. The abnormal accumulation of gallium was reported on the following criteria:

1. Any tracer accumulation in cervical, mediastinal, axillary and inguinal region was considered to be abnormal if it was seen on the 48 hour images and persistent in the delayed views.

2. Patchy and non-uniform tracer uptake in liver and spleen.
3. Any tracer accumulation in the abdomen that increased in its intensity and did not change location up to the $11^{\text {th }}$ day post IV.

4. Uptake in bones greater than or equal to liver was considered abnormal.

Equivocal sites were reported on the following criteria:

1. Tracer uptake in the neck, chest and any other lymph node regions if it was higher than the background, but poorly defined.

2. Activity in the abdomen and pelvis which changed very little in intensity and position over 5 to 11 days.

The Ga-67 uptake in the lesions was assessed using semiquantitative regions of interest to obtain 'lesion to liver ratio' (LLR) values for correlation with the histological sub-types and the results of visual grading analysis ( $>1.1$ was considered abnormal). All regions were applied to the 48 hour planar views for this analysis.

\section{Statistical Analysis}

Statistical analysis was performed using SPSS for Windows (version 10.0) software. The differences between independent means and proportions was calculated with a 95\% confidence interval (CI). Confidence intervals without an overlap and / or those which did not include zero were considered to support a statistically significant difference while confidence intervals with an overlap and/or those that included zero represented differences for which chance could not be excluded as the cause. The $F$ test analysis of variances was used to determine statistically significant differences within grouped data. A $p$-value less than 0.05 was considered significant.

\section{RESULTS}

T-cell NHL was present in $16.9 \%$ of the patients while Bcell Lymphoma was present in $76.9 \%$, where as $6.2 \%$ of the patients had anaplastic type of NHL. Gallium uptake was seen in $75.5 \%$ of the patients, while $24.5 \%$ failed to show any significant uptake. 42 patients had lymph node involvement and 16 patients had extra-nodal disease. The histopathological classification according to the REAL classification is summarized in Table 1. The most common sub-type of NHL was Diffuse Large B-cell Lymphoma.

The mean lesion to liver ratios of the various histopathological sub-types of NHL are summarized in Table 2. Table 3 shows the case number wise intensity of uptake (when compared to the liver) in the various histological subtypes of patients with nodal disease. In cases of Burkitt's lymphoma, the tumor uptake of gallium-67 was more than liver in 6 out of 10 cases of nodal NHL, and 4 out of 5 cases of extra-nodal disease (Table 4).

While there was some variation in the mean lesion to liver ratios between NHL sub-types (lymphoblastic being high at 5.4 and anaplastic $\mathrm{Ki}-1$ being low at 1.6), there was no statistically significant difference between subtypes ( $p=$ $0.521)$. This observation is supported by the overlap of the 95\% CIs. Eight of the Diffuse Large B-cell lymphoma cases had no gallium-67 uptake, where as four of that variety had intensely increased uptake. 
Table 1. REAL Staging Classification of the Study Cases

\begin{tabular}{|l|c|c|}
\hline \multicolumn{1}{|c|}{ REAL Histopathological Type } & Frequency & Percent \\
\hline \hline Anaplastic large cell lymphoma & 6 & 9.2 \\
\hline Angioimmunoblastic T-cell lymphoma & 1 & 1.5 \\
\hline $\begin{array}{l}\text { B-cell chronic lymphocytic } \\
\text { leukemia/prolymphocytic }\end{array}$ & 8 & 12.3 \\
\hline Burkitt's Lymphoma & 15 & 23.1 \\
\hline Diffuse large B-cell lymphoma & 7 & 10.8 \\
\hline Follicle centre cell lymphoma, follicular & 9 & 13.8 \\
\hline Large granular lymphocyte leukemia & 9 & 13.8 \\
\hline Lymphoplasmacytic lymphoma & 1 & 1.5 \\
\hline Mycosis fungoides/Sezary's syndrome & 1 & 1.5 \\
\hline Peripheral T-cell lymphomas, unspecified & 3 & 4.6 \\
\hline Plasmacytoma/plasma cell myeloma & 2 & 3.1 \\
\hline $\begin{array}{l}\text { Precursor B-cell neoplasm: } \\
\text { precursor B-lymphoblast }\end{array}$ & 2 & 3.1 \\
\hline $\begin{array}{l}\text { T-cell chronic lymphocytic } \\
\text { leukemia/prolymphocytic }\end{array}$ & 1 & 1.5 \\
\hline
\end{tabular}

\section{DISCUSSION}

Gallium has an established role in staging and prognosis of NHL. Baseline Ga-67 scans help in staging by localizing the nodal and extranodal sites of disease through the assessment of Ga-67 avidity. Follow-up studies aid in differentiating residual disease and fibrotic/necrotic tissue, and to assess response to therapy. Ga-67 imaging detects relapse at the earliest time and reliably predicts disease free survival.

While the most common type of NHL was Diffuse Large B-Cell lymphoma, there was a strong representation of Burkitt's type NHL (27.5\%). This aggressive type of lymphoma has a viral aetiology (i.e. the Epstein - Barr virus $[\mathrm{EBV}])$ and may reflect the increased prevalence of EBV in Pakistani population [9].

Burkitt's type of lymphoma, which is considered as one of the most aggressive variety has the highest uptake of Ga67. This might be suggestive of a difference in uptake between low- and high-grade lymphomas, although this study could not statistically validate this.

Eight of the patients with nodal Diffuse Large B-cell lymphoma cases had no gallium-67 uptake, where as 4 of that variety had intensely increased uptake, suggesting there

Table 2. Lesion to Liver Ratios of Various Histopathological Types of NHL. Overlap of the 95\% CIs Support a Lack of Statistically Significant Difference Between Means

\begin{tabular}{|l|c|c|c|c|}
\hline \multicolumn{1}{|c|}{ REAL Staging Classification } & N & Mean LLR & 95\% CI & \pm 1.85 \\
\hline \hline Burkitt's Lymphoma & 14 & 2.2408 & $\pm 5.87 *$ & \pm .5226 \\
\hline Lymphoblastic & 6 & 5.3565 & $\pm 2.00^{*}$ & \pm 1.04 \\
\hline Diffuse Large B-cell Lymphoma & 15 & 1.9961 & $\pm 2.22 *$ & - \\
\hline Peripheral T-cell and NK-cell & 3 & 2.0309 & - & 2.2692 \\
\hline Follicle Center Cell (small, large, mixed) & 4 & 1.6770 & - \\
\hline Angioimmunoblastic T-cell Lymphoma & 1 & 0.1057 & - \\
\hline Mycosis Fungoides/Sezary's Syndrome & 1 & 0.1057 & 1.9519 \\
\hline Anaplastic (Ki-1) & 4 & 1.5884 & - & - \\
\hline Anaplastic Large T-cell/CD30+/NK type & 1 & 0.1924 & - \\
\hline
\end{tabular}

*95\% CI includes zero (0) indicating chance can't be eliminated as the cause of the observed effect.

Table 3. Intensity of Nodal Uptake Compared to the Liver Uptake (Case Numbers) in Various Sub-Types of NHL

\begin{tabular}{|c|c|c|c|c|}
\hline Lymphoblastic & 5 & & & 1 \\
\hline Diffuse large B-cell lymphoma & 8 & 1 & 1 & 4 \\
\hline Follicle center cell (small, large, mixed) & 1 & 1 & 1 & 1 \\
\hline Angioimmunoblastic T-cell lymphoma & & 1 & & \\
\hline Anaplastic (Ki-1) & 1 & & & 1 \\
\hline Anaplastic Large T-cell/CD30+/NK type & 1 & & & 1 \\
\hline
\end{tabular}


Table 4. Intensity of Uptake in Extra-Nodal Disease Sites in Various Histological Sub-Types of NHL

\begin{tabular}{|c|c|c|c|c|c|}
\hline Lymphoblastic & & 1 & 1 & 1 & 3 \\
\hline Diffuse Large B-cell Lymphoma & & & 1 & 2 & 3 \\
\hline Follicle Center Cell (small, large, mixed) & 1 & & & & 1 \\
\hline Angioimmunoblastic T-Cell Lymphoma & & 1 & & & 1 \\
\hline Mycosis Fungoides/Sezary's Syndrome & & 1 & & & 1 \\
\hline Anaplastic (Ki-1) & & 1 & & & 1 \\
\hline
\end{tabular}

might be some receptor (like CD70) [9] difference in this variety that causes intense or absent uptake of Ga-67.

Our results suggest that perhaps there does not exist a direct relationship between different histopathological subtypes of NHL and Ga-67 uptake. This is probably because NHL is a heterogeneous collection of diseases with a variety of different etiologies, patterns and prognoses. Also since the Ga-67 uptake is dependent on a variety of factors, uniform uptake pattern even in a single histopathological sub-type of disease is not there [10].

The overall positivity rate of Ga- 67 scan was more than $88 \%$ in T-cell lymphomas and more than $73 \%$ in B-cell lymphomas. Lesion in high-grade/aggressive types of NHL showed a higher gallium avidity, but it was not so in every instance of the disease. Semi-quantitative grading failed to establish a relationship between Ga-67 uptake and histological sub-type. This meant that some factor in addition to the histological type was determinant of Ga-67 uptake in NHL. Further study might be warranted to confirm these results and other studies will be required to seek the determinant of Ga-67 uptake in NHL, such as transferrin or other receptors.

\section{CONCLUSION}

Gallium-67 imaging in Hon-Hodgkin's lymphoma is a reasonable and cheap alternative to positron imaging with F18 FDG, however it lacks the power to discriminate between the various histological sub-types of the disease.

\section{ACKNOWLEDGEMENT}

Declared none

\section{CONFLICT OF INTEREST}

Declared none

\section{REFERENCES}

[1] Müller AMS, Ihorst G, Mertelsmann R, Engelhardt $M$. Epidemiology of non-Hodgkin's lymphoma (NHL): trends, geographic distribution, and etiology. Ann Hematol 2005; 84: 1-12.

[2] Jemal A, Siegel R, Xu J, Ward E. Cancer Statistics, 2010. CA Cancer J Clin 2010; 60: 277-300.

[3] Even-Sapir E, Israel O. Gallium-67 scintigraphy: a cornerstone in functional imaging of lymphoma. Eur J Nucl Med Mol Imaging 2003; 30: S65-S81.

[4] The Non-Hodgkin's Lymphoma Classification Project. A Clinical Evaluation of the International Lymphoma Study Group Classification of Non-Hodgkin's Lymphoma. Blood 1997; 89: 3909-18.

[5] Chan JK, Banks PM, Cleary ML, et al. A revised EuropeanAmerican classification of lymphoid neoplasms proposed by the International Lymphoma Study Group. A summary version. Am J Clin Pathol 1995; 103: 543-60.

[6] Koeppen H, Vardiman JW. New entities, issues, and controversies in the classification of malignant lymphoma. Semin Oncol 1998; 25: 421-34.

[7] Weiner RE. The mechanism of $67 \mathrm{Ga}$ localization in malignant disease. Nuclear Med Biol 1996; 23: 745-51.

[8] Even-Sapir E, Israel O. Gallium-67 scintigraphy: a cornerstone in functional imaging of lymphoma. Eur J Nucl Med Mol Imaging 2003; 30: S65-S81.

[9] Mansoor A, Stevenson MS, Li RZ, et al. Prevalence of EpsteinBarr viral sequences and EBV LMP1 oncogene deletions in Burkitt's lymphoma from Pakistan: epidemiological correlations. Hum Pathol 1997; 28: 283-8.

[10] Tsukamoto N, Kojima M, Hasegawa M, et al. The usefulness of 18F-fluorodeoxyglucose positron emission tomography (18F-FDGPET) and a comparison of 18F-FDG-pet with 67gallium scintigraphy in the evaluation of lymphoma. Cancer 2007; 110: 652-59. 